\title{
INVARIANT MEANS ON ALMOST PERIODIC FUNCTIONS AND EQUICONTINUOUS ACTIONS
}

\author{
ANTHONY TO-MING LAU ${ }^{1}$
}

ABSTRACT. Let $S$ be a topological semigroup such that the almost periodic functions on $S$ have a left invariant mean (this is the case, for example, when $S$ has finite intersection property for closed right ideals). Then whenever $S$ acts equicontinuously on a compact Hausdorff space $X$, there exists a compact group $G$ of homeomorphisms acting equicontinuously on a retract $Y$ of $X$ such that $S$ has a common fixed point in $X$ if and only if $G$ has a common fixed point in $Y$. This result generalises some recent work of $\mathrm{T}$. Mitchell. As an application, we show that whenever $S$ acts equicontinuously on the closed unit interval $I$, then $I$ contains a common fixed point for $S$.

1. Introduction. Let $S$ be a semigroup of equicontinuous self maps of $X$, a compact Hausdorff space. Recently, T. Mitchell [6] showed that if $S$ has finite intersection property for right ideals, then there is a compact group $G$ of homeomorphisms of a retract $Y$ of $X$ with the property that $S$ has a common fixed point in $X$ if and only if $G$ has a common fixed point in $Y$. It is the purpose of this note to modify Mitchell's result. In particular, we show that the same conclusion also holds when $S$ satisfies an analytic condition (which is implied by Mitchell's algebraic condition), namely, the existence of a left invariant mean on the space of almost periodical functions on $S$.

2. Some notations. For the rest of this paper $S$ will be a fixed topological semigroup (with separately continuous multiplication).

Let $X$ be a compact Hausdorff space and let $\mathcal{U}$ be the unique uniformity on $X$. Then $S$ is said to act equicontinuously on $X$ if there exists a continuous mapping from the product space $S \times X \rightarrow X$, denoted by $(s, x)$

Received by the editors December 20,1971 and, in revised form, January 9, 1973.

AMS (MOS) subject classifications (1970). Primary 43A07, 43A60; Secondary 54H25, 54H15, 47D05, 54C30, 22A15.

Key words and phrases. Invariant means, almost periodic functions, compact groups, topological semigroups, equicontinuous mappings, common fixed points, commuting real functions.

${ }^{1}$ This research was supported by NRC Grant no. A-7679. 
$\rightarrow s \cdot x$, such that

(1) $s \cdot(t \cdot x)=(s t) \cdot x$ for all $s, t \in S$;

(2) for each $x \in S, U \in \mathcal{U}$, and $y \in X$, there exists $V \in \mathcal{U}$ such that $(x, y) \in V$ implies $(s \cdot x, s \cdot y) \in U$ for all $s \in S$.

Let $C(X, X)$ denote the space of continuous functions from $X$ to $X$ with the topology of uniform convergence on $X$, and let $\sigma: S \rightarrow C(X, X)$ be given by $(\sigma s)(x)=s \cdot x$ for all $s$ in $S$ and $x$ in $X$. Then $\bar{S}$, the closure of $S$ in $C(X, X)[3, \mathrm{p} .270]$ is a compact topological semigroup with jointly continuous multiplication.

For any topological space $Y, C(Y)$ will denote the space of bounded real-valued functions on $Y$. A function $f$ in $C(S)$ is almost periodic if $\left\{l_{a} f\right.$; $a \in S\}$ is relatively compact in the sup norm topology of $C(S)$, where $\left(l_{a} f\right)(s)$ $=f(a s)$ for all $a, s \in S$. Then, as known, $A P(S)$ the space of almost periodic functions on $S$ is translation invariant, sup norm closed and containing constants (see [2, p. 80]). An element $\phi$ in $A P(S)^{*}$, the continuous dual of $A P(S)$, is a mean if $\|\phi\|=1$ and $\phi(f) \geq 0$ whenever $f \in A P(S)$ and $f \geq 0$. If, in addition, $\phi\left(l_{a} f\right)=\phi(f)$ for all $a \in S$ and $f \in A P(S)$, then $\phi$ is a LIM (left invariant mean).

Let $\Delta(S)$ denote the set of all means $\phi$ of $A P(S)$ which are multiplicative, i.e. $\phi(f g)=\phi(f) \phi(g)$ for all $f, g \in A P(S)$. Then, as known, $\Delta(s)$ is weak*-compact and the set $\left\{p_{a} ; a \in S\right\}$, where $p_{a}(f)=f(a)$ for all $f \in A P(S)$, of point measures on $A P(S)$ is weak*-dense in $\Delta(S)$. Furthermore, the Aren's product, $(\rho \odot \mu)(f)=\rho(h)$ where $h(s)=\mu\left(l_{s} f\right)$ for all $s \in S, \rho, \mu \in \Delta(s)$ renders $\Delta(S)$ into a compact topological semigroup with the weak* topology and the multiplication in $\Delta(S)$ is even jointly continuous (see Pym $[7, \$ 5]$ ).

A topological semigroup $S$ is left reversible if any two closed right ideals in $S$ have nonempty intersection. As known, if $S$ is left reversible, then $A P(S)$ has a LIM. However, the class of topological semigroups $S$ for which $A P(S)$ has a I.IM and yet $S$ is not left reversible is huge (see for example [5, Remark 3.4]).

3. The main theorem. The proof of our main theorem is based on the following two lemmas. The first one is known.

Lemma 1 [5, Lemma 3.1]. If $S$ acts equicontinuously on a compact Hausdorff space $X$, and $x \in X$, then $T_{x} f \in A P(S)$ for any $f$ in $C(X)$, where $\left(T_{x} f(s)=f(s \cdot x)\right.$ for all $s \in S$.

Lemma 2. If $S$ acts equicontinuously on a compact Hausdorff space $X$, then $\bar{S}$ is a continuous homomorphic image of the compact semigroup $\Delta(S)$. 
Proof. For each $x$ in $X, \phi$ in $\Delta(S)$, let $(h \phi)(x)$ be a cluster point of the net $\left\{s_{a} \cdot x\right\}$ in $X$ where $\left\{p_{s}\right\}$ is a net of point measures on $A P(S)$ conveging to $\phi$ in the weak* topology. Let $\left\{p_{t}\right\}$ be another net of point measures on $A P(S)$ converging to $\phi$ in the weak* topology. By passing to subnets if necessary, we may assume that $s_{\alpha} \cdot x \rightarrow y$ and $t_{\beta} \cdot x \rightarrow z$. If $f \in C(X)$, then it follows from Lemma 1 that $T_{x} f \in A P(S)$; hence

$$
f(y)=\lim _{\alpha} f\left(s_{\alpha} x\right)=\lim _{a} p_{s_{\alpha}}\left(T_{x} f\right)=\phi\left(T_{x} f\right)=\lim _{\beta} f\left(t_{\beta} x\right)=f(z) .
$$

Therefore $h: \phi \rightarrow h \phi$ defines a mapping from $\Delta(S)$ into $\bar{S}$. It is a routine matter to verify that $h$ is a continuous homomorphism of $\Delta(S)$ onto $\bar{S}$.

A topological semigroup $S$ is said to have property $(K)$ if whenever $S$ acts equicontinuously on a compact Hausdorff space $X$, there exists a compact subgroup $G$ of $\bar{S}$ and a retract $Y$ of $X$ satisfying the following two conditions:

(a) the restriction of $G$ to $Y$ is a group of homeomorphisms from $Y$ onto $Y$;

(b) $G$ has a common fixed point in $Y$ if and only if $S$ has a common fixed point in $X$.

Theorem. If $A P(S)$ has a LIM, then $S$ has property (K).

Proof. If $A P(S)$ has a LIM, then $\Delta(S)$ is left reversible (see [2, $\$ \$ 5$, $6]$ ). Hence by Lemma $2, \bar{S}$ is also left reversible. Our result now follows from [6, Theorem 1].

Corollary 1. If $A P(S)$ has a LIM, then whenever $S$ acts equicontinuously on the closed unit interval I, I has a common fixed point for $S$.

Proof. Let $G$ be a compact subgroup of $\bar{S}$ and $Y$ a retract of $I$ with properties as stated in $(\mathrm{K})$. Since $Y$ is a closed subinterval of $I$, it follows from [6, Theorem 2] that $G$ has a common fixed point in $Y$. Hence $S$ has a common fixed point in $I$.

Corollary 2. If $S$ is left reversible, then $S$ has property $(\mathrm{K})$.

Proof. If $S$ is left reversible, then $A P(S)$ has LIM (see proof of Corollary 3.3 in [5]).

Our main theorem and the two corollaries are due to Mitchell [6] for the case when $S$ is a discrete left reversible semigroup.

An open problem. Does property $(\mathrm{K})$ imply $A P(S)$ has a LIM?

The author would like to thank the referee for his many helpful suggestions, and for his correction of an error in the original version of the paper. 


\section{REFERENCES}

1. W. M. Boyce, Commuting functions with no common fixed point, Trans. Amer. Math. Soc. 137 (1969), 77-92. MR 38 \#4627.

2. K. DeLeeuw and I. Glicksberg, Application of almost periodic compactifications, Acta Math. 105 (1961), 63-97. MR 24 \#A1632.

3. K. H. Hofmann and P. S. Mostert, Elements of compact semigroups, Merrill, Columbus, Ohio, 1966. MR 35 \#285.

4. J. P. Huneke, On common fixed points commuting continuous functions on an interval, Trans. Amer. Math. Soc. 139 (1969), 371-381. MR 38 \#6005.

5. A. Lau, Invariant means on almost periodic functions and fixed point properties, Rocky Mountain J. Math. 3 (1973), 69-76.

6. T. Mitchell, Common fixed-points for equicontinuous semigroups of mappings, Proc. Amer. Math. Soc. 33 (1972), 146-150. MR 44 \#6923.

7. J.S. Pym, The convolution of functionals on spaces of bounded functions, Proc. London Math. Soc. 15 (1965), 84-104. MR 30 \#3367.

DEPARTMENT OF MATHEMATICS, UNIVERSITY OF ALBERTA, EDMONTON 7, ALBERTA, CANADA 